TRANSACTIONS OF THE

AMERICAN MATHEMATICAL SOCIETY

Volume 275, Number 1, January 1983

\title{
ARITHMETIC EQUIVALENT OF ESSENTIAL SIMPLICITY OF ZETA ZEROS
}

BY

JULIA MUELLER

\begin{abstract}
Let $R(x)$ and $S(t)$ be the remainder terms in the prime number theorem and the Riemann-von Mangoldt formula respectively, that is $\psi(x)=x+R(x)$ and $N(t)=(1 / 2 \pi) \int_{0}^{t} \log (\tau / 2 \pi) d \tau+S(t)+7 / 8+O(1 / t)$. We are interested in the following integrals: $J(T, \beta)=\int_{1}^{T^{\beta}}(R(x+x / T)-R(x))^{2} d x / x^{2}$ and $I(T, \alpha)=$ $\int_{1}^{T}(S(t+\alpha / L)-S(t))^{2} d t$, where $L=(2 \pi)^{-1} \log T$. Furthermore, denote by $N(T, \alpha)\left(N^{*}(T)\right)$ the number of pairs of zeros $\frac{1}{2}+i \gamma, \frac{1}{2}+i \gamma^{\prime}$ with $0<\gamma \leqslant T$ and $0<\left(\gamma^{\prime}-\gamma\right) L \leqslant \alpha\left(\left(\gamma^{\prime}-\gamma\right) L=0\right)$-i.e., off-diagonal and diagonal pairs.

THEOREM. Assume the Riemann hypothesis. The following three hypotheses (A), (B) and $\left(\mathrm{C}_{1}, \mathrm{C}_{2}\right)$ are equivalent: for $\beta \rightarrow \infty$ and $\alpha \rightarrow 0$ as $T \rightarrow \infty$ we have

(A) $J(T, \beta) \sim \beta T^{-1} \log ^{2} T$,

(B) $I(T, \alpha) \sim \alpha T$ and

$\left(\mathrm{C}_{1}\right) N^{*}(T) \sim T L, \quad\left(\mathrm{C}_{2}\right) N(T, \alpha)=o(T L)$. Hypothesis $\left(\mathrm{C}_{1}, \mathrm{C}_{2}\right)$ is called the essential simplicity hypothesis.
\end{abstract}

1. Introduction. Let $R(x)$ be the remainder term in the prime number theorem when the theorem is stated as

$$
\psi(x)=x+R(x) .
$$

Let $S(T)$ be the remainder term in the Riemann-von Mangoldt zero counting formula

$$
N(T)=\frac{1}{2 \pi} \int_{0}^{T} \log \frac{t}{2 \pi} d t+S(T)+\frac{7}{8}+O\left(\frac{1}{T}\right),
$$

where $N(T)$ is the number of zeros $\beta+i \gamma$ of the Riemann zeta function with $0<\beta<1$ and $0<\gamma \leqslant T$. It is well known that the Riemann hypothesis implies that

$$
R(x)=O\left(x^{1 / 2} \log ^{2} x\right) \text { and } S(T)=O\left(\frac{\log T}{\log \log T}\right) .
$$

Furthermore, it is known that the above bound for $R(x)$ is equivalent to the truth of the Riemann hypothesis. We will assume the Riemann hypothesis throughout this paper. Cramér [1] showed, assuming the Riemann hypothesis, that

$$
\int_{1}^{X}(R(x))^{2} \frac{d x}{x^{2}} \sim C \log X, \quad C=\sum_{\gamma}^{\prime} \frac{m^{2}(\gamma)}{\left|\frac{1}{2}+i \gamma\right|^{2}},
$$

Received by the editors July 30, 1980 and, in revised form, November 23, 1981.

1980 Mathematics Subject Classification. Primary 10H05, 10H15; Secondary 10H25, 10A21.

Key words and phrases. Prime number theorem, Riemann-von Mangoldt formula, remainder term, Riemann zeta function, zeros, simple zeros, pairs of zeros, essential simplicity of zeros. 
where $m(\gamma)$ is the multiplicity of the zero $\frac{1}{2}+i \gamma$, and the dash indicates that the sum is over distinct values of $\gamma$. A. Selberg [7] showed that

$$
\int_{1}^{T}(S(t))^{2} d t \sim \pi^{-2} T \log \log T .
$$

Furthermore, he considered the following integrals:

$$
J(T, \beta)=\int_{1}^{T^{\beta}}\left(R\left(x+\frac{x}{T}\right)-R(x)\right)^{2} \frac{d x}{x^{2}}
$$

and

$$
I(T, \alpha)=\int_{1}^{T}\left(S\left(t+\frac{\alpha}{L}\right)-S(t)\right)^{2} d t,
$$

where $L=(2 \pi)^{-1} \log T$. We remark that the average spacing between consecutive zeros $\frac{1}{2}+i \gamma$ with $\gamma \leqslant T$ is asymptotic to $L^{-1}$.

Selberg [8] showed that the Riemann hypothesis implies that $J(T, \beta)$ is « $T^{-1} \log ^{2} T$, for each positive $\beta$. In fact, it is known that

$$
J(T, \beta) \begin{cases}\sim \frac{1}{2} \beta^{2} T^{-1} \log ^{2} T, & \text { for } 0<\beta \leqslant 1, \\ \ll \beta T^{-1} \log ^{2} T, & \text { for } \beta>1 .\end{cases}
$$

The above asymptotic formula can be evaluated easily by a simple method (see [4]), while the bound is due to Montgomery [5].

The following result for $I(T, \alpha)$ was first obtained by Selberg and later by A. Fujii [2]:

$$
I(T, \alpha) \begin{cases}\sim \pi^{-2} T \log \alpha, & \text { for } \alpha \rightarrow \infty, \alpha \leqslant L ; \\ \ll T \log (2+\alpha), & \text { for } \alpha \ll 1 .\end{cases}
$$

We will show in $\S 3$ that for $\alpha \rightarrow 0$,

$$
I(T, \alpha) \ll T \alpha .
$$

Before stating our main result, we will introduce some notation and definitions. Let

$$
N^{*}(T)=\sum_{0<\gamma \leqslant T}^{\prime} m^{2}(\gamma)
$$

and denote by $N(T, \alpha)$ the number of pairs of zeros $\frac{1}{2}+i \gamma, \frac{1}{2}+i \gamma^{\prime}$ with $0<\gamma \leqslant T$ and $0<\left(\gamma^{\prime}-\gamma\right) L \leqslant \alpha$. We remark that in this notation

$$
N(T)=\sum_{0<\gamma \leqslant T}^{\prime} m(\gamma) .
$$

From this definition and the Riemann-von Mangoldt formula it follows easily that

$$
\varlimsup N^{*}(T) / T L \geqslant 1 \text {. }
$$

Furthermore, Montgomery [6] has shown that

$$
N^{*}(T) \leqslant\left(\frac{4}{3}+o(1)\right) T L .
$$

As for $N(T, \alpha)$ we have the following results from [4].

$$
N(T, \alpha) \begin{cases}\sim T L \alpha & \text { for } \alpha \rightarrow \infty, \alpha \leqslant T L, \\ \ll T L \alpha & \text { for } \alpha>1, \\ \ll T L & \text { for } \alpha \leqslant 1\end{cases}
$$


Moreover, the above asymptotic formula cannot hold for $\alpha \cong 1 .^{1}$ In this range, Gallagher [3] obtained the following quantitative result for $\alpha=1$ assuming the Riemann hypothesis:

$$
\varlimsup N(T, 1) / T L \leqslant .99
$$

and

$$
\underline{\lim } N(T, 1) / T L \geqslant .14 \text {. }
$$

We remark that H. L. Montgomery [6] has conjectured that

$$
N(T, \alpha) \sim T L \int_{0}^{\alpha}\left\{1-\left(\frac{\sin \pi a}{\pi a}\right)^{2}\right\} d a
$$

as $T \rightarrow \infty$, uniformly in each interval $0<\alpha_{0} \leqslant \alpha \leqslant \alpha_{1}<\infty$. We have shown in [4, pp. 206-208] that (4') implies that for $\beta \cong 1$ and $\alpha \cong 1$,

$$
J(T, \beta) \sim T^{-1} \log ^{2} T \min \left\{\beta-\frac{1}{2}, \frac{1}{2} \beta^{2}\right\}
$$

and

$$
I(T, \alpha) \sim T \int_{-1}^{1}\left(\frac{\sin \pi b}{\pi b}\right)^{2}|b| d b .
$$

For $\beta \rightarrow \infty$ and $\alpha \rightarrow 0$ we obtained
(A) $J(T, \beta) \sim \beta T^{-1} \log ^{2} T$ and
(B) $I(T, \alpha) \sim \alpha T$.

In this note we will show that hypotheses (A) and (B) are equivalent. Moreover, they are equivalent to the essential simplicity hypothesis:

$\left(\mathrm{C}_{1}\right) N^{*}(T) \sim T L$

$\left(\mathrm{C}_{2}\right) N(T, \alpha)=o(T L)(\alpha \rightarrow 0$ as $T \rightarrow \infty)$.

Theorem 1. Assume the Riemann hypothesis. The three hypotheses (A), (B) and $\left(\mathrm{C}_{1}, \mathrm{C}_{2}\right)$ are equivalent.

The meaning of essential simplicity hypothesis is that the zeros $\rho=\frac{1}{2}+i \gamma$ with $\gamma \leqslant T$ are almost all simple zeros; moreover, "nearby" zero pairs are few.

2. Lemmas. Let

$$
A(T)=\sum_{\gamma>0}^{\prime} a(\gamma) m(\gamma) \text { and } A^{*}(T)=\sum_{\gamma>0}^{\prime} a(\gamma) m^{2}(\gamma)
$$

where

$$
a(t)=a(t, T)=\left(\frac{\sin t / 2 T}{t / 2 T}\right)^{2} .
$$

The sums, here and later, are over distinct values of $\gamma$. We remark that from now on the symbols $T_{0}$ and $T_{1}$ will mean $T_{0}=T_{0}(T)$ such that $T_{0} / T \rightarrow 0$ and $T_{1}=T_{1}(T)$ such that $T_{1} / T \rightarrow \infty$ as $T \rightarrow \infty$.

\footnotetext{
${ }^{1}$ By $f \cong g$ we mean $f \ll g$ and $g \ll f$.
} 
LEMMA 1. We have

$$
A(T) \sim \frac{1}{2} T \log T .
$$

Moreover, $\left(\mathrm{C}_{1}\right)$ is equivalent to

$$
A^{*}(T) \sim \frac{1}{2} T \log T
$$

Proof. First we note that for each $T_{0}$

$$
\sum_{\gamma \leqslant T_{0}}^{\prime} a(\gamma) m(\gamma) \leqslant \sum_{\gamma \leqslant T_{0}}^{\prime} a(\gamma) m^{2}(\gamma) \ll T_{0} L=o(T L) .
$$

Next, for each $T_{1}$ the partial sums of $A(T)$ and $A^{*}(T)$ of the range $\left[2^{k} T_{1}, 2^{k+1} T_{1}\right]$ are

$$
\ll\left(\frac{T}{2^{k} T_{1}}\right)^{2} N\left(2^{k+1} T_{1}\right) \ll 2^{-k+1} T_{1}^{-1} T^{2} L=o\left(2^{-k+1} T L\right) .
$$

Summing over $k$, we get

$$
\sum_{\gamma>T_{1}}^{\prime} a(\gamma) m(\gamma) \leqslant \sum_{\gamma>T_{1}}^{\prime} a(\gamma) m^{2}(\gamma)=o(T L) .
$$

From the Riemann-von Mangoldt formula $N(T) \sim T L$, we get

$$
A(T) \sim \sum_{T_{0}<\gamma \leqslant T_{1}}^{\prime} a(\gamma) m(\gamma)=\frac{1}{2 \pi} \int_{T_{0}}^{T_{1}} a(t) \log \frac{t}{2 \pi} d t+\int_{T_{0}}^{T_{1}} a(t) d(o(T \log T)) .
$$

The first integral is asymptotic to

$$
T L \int_{T_{0} / T}^{T_{1} / T}\left(\frac{\sin \frac{1}{2} \theta}{\frac{1}{2} \theta}\right)^{2} d \theta \sim T L \int_{0}^{\infty}\left(\frac{\sin \frac{1}{2} \theta}{\frac{1}{2} \theta}\right)^{2} d \theta=\frac{1}{2} T \log T,
$$

provided

$$
\log T_{0} \sim \log T_{1} \sim \log T
$$

The remainder integral is

$$
o(T \log T)+o(T \log T) \int_{T_{0}}^{T_{1}}\left|a^{\prime}(t)\right| d t=o(T \log T),
$$

since $a^{\prime}(t) \ll T^{-1}(1+t / T)^{-2}$ and

$$
\int_{T_{0} / T}^{T_{1} / T}(1+t / T)^{-2} d(t / T) \leqslant \int_{0}^{\infty}(1+\theta)^{-2} d \theta=1 .
$$

This proves (5).

An entirely similar method will also show that $\left(C_{1}\right)$ implies (6). To show the converse, we first remark that from (5) and (6) we get

$$
A^{*}(T)-A(T)=o(T L) \text {. }
$$

Since $a(\gamma) \gg 1$ for $\gamma \leqslant T$ and the sum is nonnegative, it follows that

$$
N^{*}(T)-N(T)=\sum_{0<\gamma \leqslant T}\left(m^{2}(\gamma)-m(\gamma)\right)=o(T L),
$$

from which we obtain $\left(C_{1}\right)$. This completes the proof of Lemma 1. 
For each $\beta$, define

$$
A(T, \beta)=\sum_{\substack{\gamma>0 \\\left(\gamma^{\prime}-\gamma\right) L>0}} a(\gamma) S\left(\left(\gamma^{\prime}-\gamma\right) L \beta\right)
$$

where

$$
S(b)=\left(\frac{\sin \pi b}{\pi b}\right)^{2} .
$$

We remark that from now on the symbol $\beta$ will mean $\beta=\beta(T)$ such that $\beta \rightarrow \infty$ as $T \rightarrow \infty$.

LEMMA 2. The hypothesis $\left(\mathrm{C}_{2}\right)$ is equivalent to

$$
A(T, \beta)=o(T L) \quad(\beta \rightarrow \infty \text { as } T \rightarrow \infty) .
$$

Proof. First we will show that

$$
\sum_{\substack{\gamma>T_{1} \\\left(\gamma^{\prime}-\gamma\right) L>0}} a(\gamma) S\left(\left(\gamma^{\prime}-\gamma\right) L \beta\right)=o(T L)
$$

provided $T_{1} / T \rightarrow \infty$ as $T \rightarrow \infty$.

For each fixed $\alpha>1$, the sum over the range $2^{k} T_{1}<\gamma \leqslant 2^{k+1} T_{1}$ and $0<$ $\left(\gamma^{\prime}-\gamma\right) L \leqslant \alpha$ is

$$
\ll\left(\frac{T}{2^{k} T_{1}}\right)^{2} \cdot 2^{k+1} T_{1} \alpha L=o\left(2^{-k+1} T L\right) ;
$$

here we have used the bound for $N(T, \alpha)$ given by (4). Similarly, over the range $2^{k} T_{1}<\gamma \leqslant 2^{k+1} T_{1}$ and $2^{l} \alpha<\left(\gamma^{\prime}-\gamma\right) L \leqslant 2^{l+1} \alpha$, the sum is

$$
\ll\left(\frac{T}{2^{k} T_{1}}\right)^{2}\left(\frac{1}{2^{l} \alpha}\right)^{2} \cdot 2^{k+l+2} T_{1} \alpha L=o\left(2^{-k-l+2} T L\right) .
$$

Summing on $k \geqslant 0$ and $l \geqslant 0$, we get (8). Now we have

$$
A(T, \beta)=A_{1}(T, \beta)+o(T L) \text {, }
$$

where

$$
A_{1}(T, \beta)=\sum_{\substack{0<\gamma \leq T_{1} \\\left(\gamma^{\prime}-\gamma\right) L>0}} a(\gamma) S\left(\left(\gamma^{\prime}-\gamma\right) L \beta\right) .
$$

To prove Lemma 2 , it suffices to show that $\left(\mathrm{C}_{2}\right)$ is equivalent to

$$
A_{1}(T, \beta)=o(T L)
$$

provided $T_{1} / T \rightarrow \infty$ sufficiently slowly.

We remark that for $0<\gamma \leqslant T$ and $0<\left(\gamma^{\prime}-\gamma\right) L \leqslant \alpha$ each term in the sum of $A_{1}(T, \beta)$ over this range is $\gg 1$ provided $\alpha \leqslant(2 \beta)^{-1}$. Since $A_{1}(T, \beta)$ is a sum of nonnegative terms we have

$$
N(T, \alpha) \ll A_{1}(T, \beta) .
$$

Hence $\left(C_{2}\right)$ follows from (10). 
To show the converse, we split $A_{1}(T, \beta)$ into two parts, $\Sigma^{\prime}$ and $\Sigma^{\prime \prime}$, according as $0<\left(\gamma^{\prime}-\gamma\right) L \leqslant \alpha$ or $\left(\gamma^{\prime}-\gamma\right) L>\alpha$ where $\alpha<1$ will be determined later. We observe that if $T_{1} / T \rightarrow \infty$ sufficiently slowly then we may replace $T$ by $T_{1}$ in $\left(\mathrm{C}_{2}\right)$. Since

$$
\Sigma^{\prime} \ll N\left(T_{1}, \alpha\right),
$$

we get $\Sigma^{\prime}=o(T L)$ from $\left(\mathrm{C}_{2}\right)$. To deal with $\Sigma^{\prime \prime}$, we note that the partial sum over the range $2^{\prime} \alpha<\left(\gamma^{\prime}-\gamma\right) L \leqslant 2^{l+1} \alpha$ is

$$
\ll\left(2^{l} \alpha \beta\right)^{-2} N\left(T_{1}, 2^{l+1} \alpha\right) \ll \begin{cases}2^{-l+1} \alpha^{-1} \beta^{-2} T_{1} L & \text { for } 2^{l+1} \alpha \geqslant 1, \\ 2^{-2 l}(\alpha \beta)^{-2} T_{1} L & \text { otherwise. }\end{cases}
$$

Suppose that $(\alpha \beta)^{-1} \rightarrow 0$ and $T_{1} / T \rightarrow \infty$ sufficiently slowly as $T \rightarrow \infty$; then on summing over $l \geqslant 0$, we get

$$
\Sigma^{\prime \prime}=o(T L)
$$

This proves Lemma 2.

LEMMA 3. We have

$$
\int_{0}^{\beta} J(T, b) d b=\beta^{2} T^{-2} \log T\left\{A^{*}(T)+2 A(T, \beta)+o(T L)\right\}
$$

where $\beta=\beta(T)$ such that $\beta \rightarrow \infty$ as $T \rightarrow \infty$.

Proof. We begin by quoting the following result from $\$ 3$ of [4]: for each fixed $\beta>0$,

$$
J(T, \beta)=T^{-2} \log T\left\{\int_{0}^{\beta}\left(2 \operatorname{Re} \sum c(\gamma) e(\gamma L b)\right)^{2} d b+o(T L)\right\}
$$

where

$$
c(\gamma)=\frac{1-e^{i \gamma / T}}{i \gamma / T}
$$

and the remainder term is independent of $\beta$. We remark that (13) also holds provided $\beta \rightarrow \infty$ sufficiently slowly.

We will show that after smoothing the integral in (13), its partial sum over $\gamma>T_{1}$ is negligible; therefore the remaining part of that double integral corresponds to terms on the right-hand side of (12). That is

$$
\int_{0}^{\beta} \int_{0}^{b}\left(2 \operatorname{Re} \sum_{\gamma>T_{1}} c(\gamma) e(\gamma L w)\right)^{2} d w d b=o(\beta T L) \quad(\beta \rightarrow \infty \text { as } T \rightarrow \infty)
$$

and

$$
\int_{0}^{\beta} \int_{0}^{h}\left(2 \operatorname{Re} \sum_{0<\gamma \leqslant T_{1}} c(\gamma) e(\gamma L w)\right)^{2} d w d b=\beta^{2}\left\{A^{*}(T)+2 A(T, \beta)+o(T L)\right\} .
$$


First we remark that the integral in (14) is

$$
\begin{aligned}
& \ll \int_{-\beta}^{\beta}\left(1-\frac{|b|}{\beta}\right)\left|\sum_{\gamma>T_{1}} c(\gamma) e(\gamma L b)\right|^{2} d b \\
& \ll L^{-1} \int_{-\beta L}^{\beta L}\left|\sum_{\gamma>T_{1}} c(\gamma) e(\gamma y)\right|^{2} d y \quad(y=L b) .
\end{aligned}
$$

Using the same method as that in $\$ 3$ of [4] for bounding means of exponential sums, the last integral can be shown to be

$$
\ll \int_{\frac{1}{2} T_{1}}^{\infty}\left(\frac{1}{U} \sum_{t}^{t+U}|c(\gamma)|\right)^{2} d t \ll\left(\frac{T}{U}\right)^{2} \int_{\frac{1}{2} T_{1}}^{\infty}\left(\Delta_{U} N\right)^{2} \frac{d t}{t^{2}}
$$

where

$$
\begin{gathered}
\Delta_{U} N(t)=N(t+U)-N(t), \\
U L=\alpha=(2 \beta)^{-1} \text { and } c(t) \ll T / t .
\end{gathered}
$$

We assert that the contribution of the last integral from the interval $\left(2^{j} T_{1}, 2^{j+1} T_{1}\right]$ is $\ll\left(2^{j} T_{1}\right)^{-1}(U(j+L))$. Summing on $j \geqslant-1$, we get a bound

$$
\ll\left(T_{1} \alpha\right)^{-1} T^{2} L=o(\beta T L) .
$$

The assertion is a consequence of the bounds (3) and (4) for $N^{*}(T)$ and $N(T, \alpha)$ for $\alpha \rightarrow 0$ and the formula that has been proved on p. 209 of [4]:

$$
\int_{T}^{2 T}\left(\Delta_{U} N\right)^{2} d t \leqslant U N^{*}(T)+2 \int_{0}^{\alpha} N(T, a) d a+O\left(L^{2}\right) .
$$

Next, we note that (15) may be written as

$$
\begin{aligned}
\beta^{2}\left\{\sum_{0<\gamma \leqslant T_{1}} a(\gamma) m^{2}(\gamma)+2 \sum_{\substack{0<\gamma \leqslant T_{1} \\
\left(\gamma^{\prime}-\gamma\right) L>0}} \overline{c(\gamma)} c\left(\gamma^{\prime}\right) S\left(\left(\gamma^{\prime}-\gamma\right) L \beta\right)\right. & \\
& \left.+\sum_{0<\gamma, \gamma^{\prime} \leqslant T} c(\gamma) c\left(\gamma^{\prime}\right) S\left(\left(\gamma^{\prime}+\gamma\right) L \beta\right)\right\},
\end{aligned}
$$

since

$$
\begin{aligned}
& \int_{0}^{\beta} \int_{0}^{b} 2 \operatorname{Re} e(a w) d w d b=\left(\frac{\sin \pi a \beta}{\pi a}\right)^{2}=\beta^{2} S(a \beta), \\
& (2 \operatorname{Re} S)^{2}=2|S|^{2}+2 \operatorname{Re}\left(S^{2}\right) \text { and } a(\gamma)=|C(\gamma)|^{2} .
\end{aligned}
$$

The first sum in the bracket is asymptotic to $A^{*}(T)$ and therefore to derive (15) it remains to show the estimates

$$
\sum_{\substack{0<\gamma \leqslant T_{1} \\\left(\gamma^{\prime}-\gamma\right) L>0}} \overline{c(\gamma)} c\left(\gamma^{\prime}\right) S((\gamma-\gamma) L \beta)-A(T, \beta)=o(T L)
$$


and

$$
\sum_{0<\gamma, \gamma^{\prime} \leqslant T_{1}} c(\gamma) c\left(\gamma^{\prime}\right) S\left(\left(\gamma^{\prime}+\gamma\right) L \beta\right)=o(T L) .
$$

We remark first that, in (17), $A(T, \beta)$ may be replaced by $A_{1}(T, \beta)$ because of (9). Also the partial sum in (17) over the range $\left(\gamma^{\prime}-\gamma\right) L>\alpha$ is $o(T L)$ provided that $\alpha$ satisfies the property assumed in (11). This can be shown in a similar way to (11). Therefore the left-hand side of (17) may be replaced by

$$
\sum_{\substack{0<\gamma \leqslant T_{1} \\ 0<\left(\gamma^{\prime}-\gamma\right) L \leqslant \alpha}} \overline{c(\gamma)}\left(c\left(\gamma^{\prime}\right)-c(\gamma)\right) S\left(\left(\gamma^{\prime}-\gamma\right) L \beta\right)+o(T L) .
$$

But the above sum is $\ll T_{1} \alpha L$, which is $o(T L)$ provided $T_{1} / T \rightarrow \infty$ sufficiently slowly.

To show (18), we let $T_{0} L=o\left(T_{1} L\right)^{1 / 2}$ and again we divide the sum into two parts $\Sigma_{1}$ and $\Sigma_{2}$, according as $0<\gamma, \gamma^{\prime} \leqslant T_{0}$ or otherwise. Assuming $T_{1} / T \rightarrow \infty$ sufficiently slowly, then

$$
\Sigma_{1} \ll N\left(T_{0}\right)^{2}=o(T L)
$$

and

$$
\Sigma_{2} \ll\left(T_{0} L \beta\right)^{-2} N\left(T_{1}\right)^{2}=o(T L) .
$$

This proves (18) and also completes the proof of Lemma 3.

3. Proof of (2) and Theorem 1.

Proof OF (2). We will first prove the formula

$$
U N^{*}(T)+2 \int_{0}^{\alpha} N(T, a) d a=I(T, \alpha)+o(T \alpha)
$$

for $\alpha \rightarrow 0$ and $T \rightarrow \infty$.

From the Riemann-von Mangoldt formula in $\S 1$, we get

$$
\begin{aligned}
\int_{T}^{2 T}\left(\Delta_{U} N\right)^{2} d t= & \int_{T}^{2 T}\left(\Delta_{U} M\right)^{2} d t+2 \int_{T}^{2 T}\left(\Delta_{U} M\right)\left(\Delta_{U} S\right) d t \\
& +\int_{T}^{2 T}\left(\Delta_{U} S\right)^{2} d t+O(L) .
\end{aligned}
$$

Since

$$
\Delta_{U} M(t) \sim \alpha
$$

for $T \leqslant t \leqslant 2 T$ and $T \rightarrow \infty$, we have

$$
\int_{T}^{2 T}\left(\Delta_{U} M\right) d t \sim \alpha^{2} T
$$

To estimate the second integral on the right-hand side of (20) we use the bound $S(T) \ll L$ to get

$$
\int_{T}^{2 T}\left(\Delta_{U} S\right)(t) d t=\int_{2 T}^{2 T+U} S(t) d t-\int_{T}^{T+U} S(t) d t \ll \alpha .
$$

Since $\Delta_{U} M$ is monotonic and $\ll \alpha$, it follows that

$$
\int_{T}^{2 T}\left(\Delta_{U} M\right)\left(\Delta_{U} S\right) d t \ll \alpha^{2}
$$


Combining (20), (21) and (22) we get, for $T \rightarrow \infty$ and $\alpha \rightarrow 0$,

$$
\int_{T}^{2 T}\left(\Delta_{U} N\right)^{2} d t=I(T, \alpha)+o(T \alpha) .
$$

From (16) and (23) we get (19), and (2) follows from (19) and the bounds (3) and (4).

Proof of Theorem 1. It is obvious that the equivalence of hypotheses (B) and $\left(C_{1}, C_{2}\right)$ is an immediate consequence of (19). Now we assume that (A) holds; then from (1) we get for $\beta \rightarrow \infty$

$$
\int_{0}^{\beta} J(T, b) d b \sim \frac{1}{2} \beta^{2} T^{-1} \log ^{2} T \quad(T \rightarrow \infty) .
$$

Combining this with (12) and using Lemmas 1 and 2, we get both $\left(\mathrm{C}_{1}\right)$ and $\left(\mathrm{C}_{2}\right)$. Conversely, suppose both $\left(C_{1}\right)$ and $\left(C_{2}\right)$ hold; then (12) simplifies to (24). Since $J(T, \beta)$ is a positive increasing function of $\beta$, we have

$$
(\beta \varepsilon)^{-1} \int_{\beta(1-\varepsilon)}^{\beta} J(T, b) d b \lesssim J(T, \beta) \lesssim(\beta \varepsilon)^{-1} \int_{\beta}^{\beta(1+\varepsilon)} J(T, b) d b
$$

for each $\varepsilon>0$. But the integrals here are asymptotic to $\beta^{2} \varepsilon\left(1 \pm \frac{1}{2} \varepsilon\right) T^{-1} \log ^{2} T$. By letting $\varepsilon \rightarrow 0$, we get (A).

\section{REFERENCES}

1. H. Cramér, Ein Mittelwertsatz in der Primzahltheorie, Math. Z. 12 (1922), 147-153.

2. A. Fujii, On the zeros of Dirichlet L-functions. I. Trans. Amer. Math. Soc. 196 (1974), 225-235.

3. P. X. Gallagher, Pair correlation of zeros of the zeta function (to appear).

4. P. X. Gallagher and J. Mueller, Primes and zeros in short intervals, J. Reine Angew. Math. 303/304 (1977), 205-220.

5. H. L. Montgomery, Gaps between primes (unpublished).

6. The pair correlation of zeros of the zeta function, Proc. Sympos. Pure Math., vol. 24, Amer. Math. Soc., Providence, R. I., 1973, pp. 181-193.

7. A. Selberg, Contributions to the theory of the Riemann zeta-function, Arch. Math. Naturvid. 48 (1946), 89-155.

8. $\ldots$, On the normal density of primes in short intervals and the difference between consecutive primes, Arch. Math. Naturvid. B 47 (1943).

Department of Mathematics, Fordham University, BronX, New YORK 10458

Current address: School of Mathematics, Institute for Advanced Study, Princeton, New Jersey 08540 